\title{
Fotografía 2D en el Diagnóstico de Asimetrías Faciales
}

\author{
2D Photography in Facial Asymmetry Diagnosis
}

S. Olate*; M. Cantín ${ }^{* *} ;$ B. Vásquez ${ }^{* * *} ;$ M. Muñoz ${ }^{* * * * * * * * *}$ \& M. de Moraes ${ }^{* * * * * *}$

OLATE, S.; CANTÍN, M.; VÁSQUEZ, B.; MUÑOZ, M. \& DE MORAES, M. Fotografía 2D en el diagnóstico de asimetrías faciales. Int. J. Morphol., 33(4):1483-1486, 2015.

RESUMEN: El objetivo de esta investigación es identificar la relación entre la asimetría facial y la pérdida de contacto en línea mediana entre dientes maxilares y mandibulares. Se realizó un estudio descriptivo en 18 sujetos portadores de asimetría facial que consultaron en la División de Cirugía Oral y Maxilofacial de la Universidad de La Frontera por tratamiento quirúrgico de la asimetría. En todos ellos se evaluó la presencia y grado de la asimetría facial y la presencia y desvío desde la línea mediana facial del punto mediano mandibular y maxilar mediante la prueba estadística t de student considerando un valor de $\mathrm{p}<0,05$; todas las evaluaciones fueron realizadas mediante fotografías $2 \mathrm{D}$ de rostro y dientes obtenidas en condiciones estandarizadas. Se identificó que todos los sujetos portadores de asimetría facial presentaron asimetría dentaria; los desvíos dentarios fueron de casi 7 mm y existió relación estadísticamente significativa entre desvío facial y desvío dentario. Se concluye que los sujetos con asimetría dentaria pueden presentar asimetría facial de forma que la evaluación esqueletal del rostro es necesaria para realizar un diagnóstico correcto.

PALABRAS CLAVE: Fotografía; Asimetría facial; Asimetría dental.

\section{INTRODUCCIÓN}

Los métodos diagnósticos en pacientes con anomalías del esqueleto facial se han desarrollado en los últimos años junto al análisis clínico, fotográfico e imagenológico. El sustento de estos análisis esta en la necesidad de un método de diagnóstico certero junto a la obtención de elementos y registros que permitan la planificación del tratamiento (Nord et al., 2015).

La asimetría facial subclínica es común y se puede considerar dentro de los parámetros de normalidad; esta asimetría se convierte en patológica cuando presenta alteraciones funcionales y cosméticas que implican dolor en la función, modificaciones de la dinámica de función (desencadenando cambios adaptativos y degenerativos de la morfología normal) y alteraciones psicosociales ocasionadas en parte por la anomalía de la forma facial (Olate et al., 2013a).

La deformidad facial asimétrica presenta componentes tridimensionales que exigen un diagnóstico certero y la evaluación la modificación terapéutica en base a cirugía de articulación temporo mandibular, cirugía ortognática o cirugía ortofacial (Olate et al., 2014). Usualmente, los sujetos con asimetría facial consultan inicialmente debido a alteraciones dentarias, determinando que esta consulta sea con el especialista en ortodoncia. En estas condiciones, el énfasis exclusivo en relaciones dentarias puede llevar a no realizar el diagnóstico correcto que incluye la anomalía esqueletal como causa de la anomalía dental.

La calidad de la fotografía para el análisis facial se ha optimizado sustancialmente en los últimos años. Ettorre et al. (2006) y Schaaf et al. (2006) desarrollaron un protocolo para la adquisición de fotografías que sugiere el uso de posición frontal, tres cuartos, lateral, entre otras, lo cual permite un análisis de la deformidad en diferentes niveles. La captura fotográfica 3D creó un nuevo tipo de análisis consiguiendo la definición de las características faciales en cualquier dimensión necesaria (Nord et al.; Nanda et al., 2015).

Division of Oral and Maxillofacial Surgery, Universidad de La Frontera, Temuco, Chile.

** CIMA Research Group, Faculty of Dentistry, CEMyQ, Universidad de La Frontera, Temuco, Chile.

**** Universidad de Tarapacá, Arica, Chile.

***** Center for Biomedical Research, Universidad Autónoma de Chile, Temuco, Chile.

****** Fellow Research, Universidad Científica del Sur, Lima, Perú.

${ }^{* * * * * * *}$ Division of Oral and Maxillofacial Surgery, Campinas State University, Campinas, Brasil.

Financiado por el Convenio Universidad es Territorio (UNETE), Proyecto UNT15-0008, Universidad de La Frontera, Temuco, Chile. 
Sin embargo, los elevados costos de los sistemas de captura fotográfica 3D impiden que sean utilizados ampliamente en el mundo; las fotografías 2D continúa siendo un elemento altamente utilizados en el diagnóstico, la planificación quirúrgica y en el análisis de resultados.

El objetivo de esta investigación fue relacionar la asimetría facial observada en fotografías bidimensionales y la condición dentaria observada en el mismo tipo de fotografía.

\section{MATERIAL Y MÉTODO}

Se diseñó un estudio de corte transversal para analizar la relación entre la fotografía 2D del rostro y las condiciones dentarias observadas en sujetos que consultaron por tratamiento quirúrgico para tratar la anomalía esqueletal.

Se incluyeron sujetos con asimetría facial e indicación terapéutica mediante cirugía ortognática u ortofacial. La asimetría facial se definió cuando, clínicamente, el punto mentón estuviera desviado sobre $5 \mathrm{~mm}$ desde la línea mediana facial (unión entre puntos glabela, pronasal, filtrum); bajo esta condición de asimetría se incluyeron pacientes con anomalías sagitales de clase I, II o III sin exclusión de anomalías de tipo vertical. Se excluyeron sujetos con patología oncológica o con antecedentes de trauma facial previo y pacientes con cirugía facial previa.

La adquisición de fotografías 2D siguió el protocolo de Schaaf et al. y Ettorre et al., identificando un punto focal (nasion) en la región facial anterior. La cámara fotográfica que se utilizó fue de marca Canon EOS Rebel t5 (Canon Inc., Tokio, Japón). La distancia entre cámara fotográfica y sujeto fue de $2 \mathrm{~m}$, considerando las condiciones de luz estandarizadas sensibilidad ISO 100 y lente macro de 105 $\mathrm{mm}$. La fotografía frontal se realizó 3 veces, estableciendo la posición natural de la cabeza con visión horizontal y plano bipupilar paralelo al piso.

Una segunda fotografía (captura realizada en tres momentos diferentes) fue realizada a los dientes, estableciendo la posición de máxima intercuspidación (PMI); el punto focal fue el punto de contacto entre los incisivos centrales superiores. En esta fotografía se analizó la posición dentaria y la relación entre la línea presente ente los incisivos centrales superiores e inferiores, estableciendo el desvío de la línea mediana presente (Fig. 1).

De las tres imágenes obtenidas en cada registro se escogió aquella que presentara mayor homogeneidad y características; la simetría de la captura fotográfica se mantu-

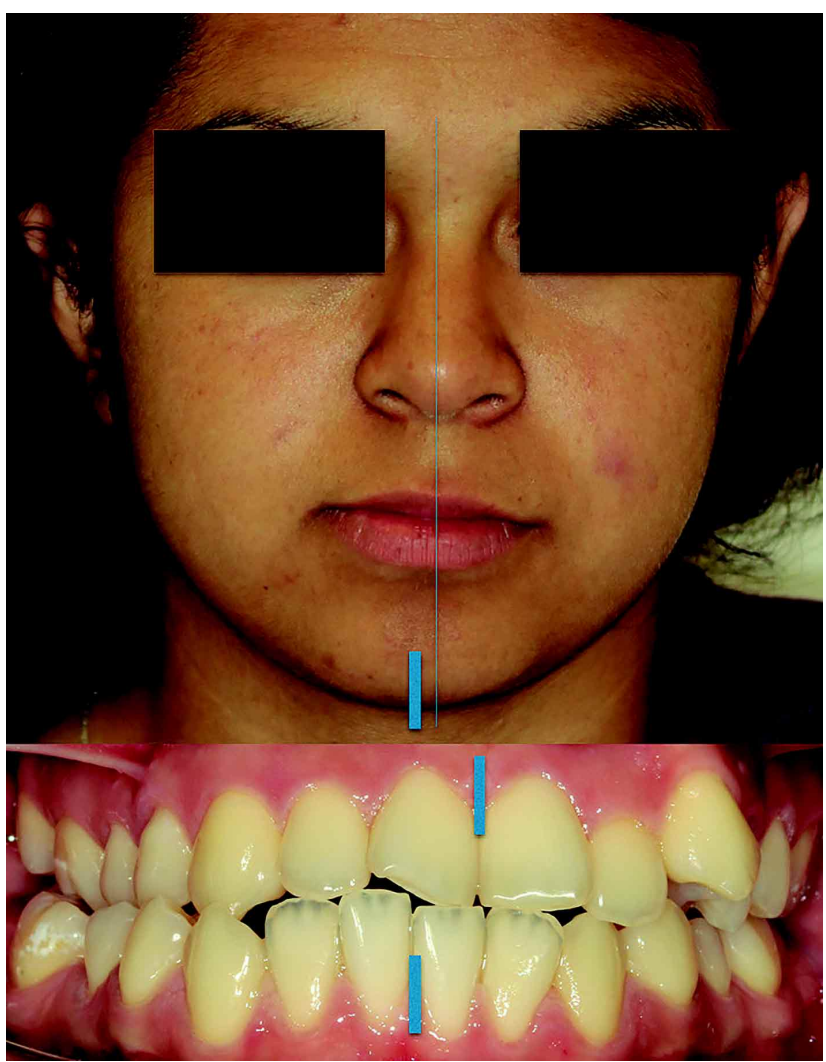

Fig. 1. Fotografía 2D obtenida de un sujeto con asimetría facial. Se determina la línea mediana facial, línea de mentón y líneas incisivas mandibular y maxilar.

vo considerando exposición de oreja externa y distancia intercantal e inter pupilar; la selección de la fotografía fue realizada por dos sujetos de forma independiente con al menos 5 años de experiencia en el diagnóstico de deformidades faciales. Las imágenes fueron posteriormente analizadas en el software Adobe Photoshop, Elements 11.0 Editor (Adobe System Inc., USA), incorporando la fotografía para realizar estudios de posición del mentón en relación a la línea mediana facial y la angulación obtenida con al comisura de los labios. El análisis de datos se realizó con la prueba estadística t de student considerando un valor de $\mathrm{p}$ $<0,05$ para considerar significancia estadística.

\section{RESULTADOS}

Fueron incluidos en esta investigación 18 sujetos de ambos sexos con una edad promedio de $19( \pm 4,53)$ años. Diez sujetos pertenecieron al grupo femenino y 8 sujetos al grupo masculino; no existió diferencia significativa entre sexo en términos de grado de asimetría facial o grado de alteración dentaria. Todos ellos presentaban deformidad facial asimétrica con indicación de tratamiento orto-quirúrgico. 
Las fotografías revelaron la presencia de asimetría facial con diferentes grados de evolución. Se observó un promedio de asimetría facial de 7,1 mm (incluyendo ambos sectores derecho e izquierdo), con máximos de $9,5 \mathrm{~mm}$ y mínimos de $6 \mathrm{~mm}$. En todos los casos se confirmó la pérdida de simetría entre los dientes, demostrando desvío de dientes mandibulares desde la línea mediana del rostro con un promedio de 5,2 $\mathrm{mm}$. La relación entre ambas condiciones fue estadísticamente significativa $(\mathrm{p}<0,05)$, determinando que la alteración dentaria se relacionó con la asimetría facial.

Los dientes mandibulares o maxilares presentaron diferentes grados de mal posición, con discrepancias negativas y formas de arco maxilar y mandibular que no fueron consideradas en esta investigación. La clase dentaria según la relación de Angle no presentó diferencias significativas en los grupos de análisis.

\section{DISCUSIÓN}

La fotografía clínica ha sido utilizada ampliamente en cirugía facial. Ettorre et al. y Schaaf et al. identificaron modelos para el desarrollo de la fotografía facial a fin de estandarizar el proceso; la fotografía clínica 2D contribuye con el diagnóstico clínico y con la educación al paciente en casos de alteraciones morfológicas.

La fotografía clínica es parte de los métodos de diagnóstico y registro utilizados en el análisis de pacientes con o sin anomalías del esqueleto facial (Huentequeo-Molina et al., 2013; Supit et al., 2015). Importante resultado de esta investigación es la relación positiva, en el estudio fotográfico 2D, entre la asimetría dentaria y la asimetría facial. Si bien esta condición puede ser esperable en ciertos grupos de anomalías esqueletales, como los sujetos con hiperplasia condilar unilateral activa (Muñoz et al., 2014; Goulart et al., 2015), la información de esta investigación se puede confirmar que la pérdida de línea mediana interdentaria se relaciona con asimetría mandibular y junto a ello con una posible deformidad del esqueleto facial.

Los análisis de la asimetría facial se han realizado con diferentes elementos morfométricos. Kim et al., (2003) realizó un estudio sobre cráneos asimétricos identificando puntos que podrían ser útiles en las mediciones de la asimetría. Song et al. (2007) definieron líneas y ángulos que pudiesen ser utilizados en el diagnóstico de asimetrías faciales mediante fotografías 2D. Maeda et al. (2006), por otra parte, indicaron que los estudios con tomografías computadorizadas podrían reemplazar los estudios 2D en el diagnóstico de asimetrías faciales. Finalmente, Gateno et al. (2011) identificaron el diagnóstico de asimetrías faciales en estudios 2D y 3D definiendo la posibilidad de diagnóstico de asimetrías sutiles mediante los dos exámenes de forma independiente.

Del punto de vista dental, la diferencia entre líneas medias interincisivas y la oclusión invertida posterior unilateral o la mordida abierta posterior unilateral también permite definir la presencia de asimetría facial (Proffit \& Turvey, 2003). Estudios previos de nuestro grupo ya determinaron alguna relación entre desvío de mentón desde la línea mediana facial y algunas alteraciones dentarias en sujetos con hiperplasia condilar activa unilateral (Olate et al., 2013b). Clínicamente es posible realizar esta observación; fotográficamente, nuestros resultados reportan que la pérdida de línea media interdentaria se relaciona con la asimetría facial.

Finalmente, podemos concluir que la consulta inicial de un sujeto que presenta pérdida de línea mediana dentaria inter maxilar implica la necesidad de observación del rostro debido a una posible asimetría facial.

\section{AGRADECIMIENTOS}

Los autores agradecen a la Universidad de La Frontera, el Ministerio de Educación, el Gobierno Regional de La Araucanía, en colaboración con otras instituciones públicas y organismos internacionales por el Convenio Universidad es Territorio (UNETE) y el apoyo al Proyecto UNT15-0008, Universidad de La Frontera, Temuco, Chile.

OLATE, S.; CANTÍN, M.; VÁSQUEZ, B.; MUÑOZ, M. \& DE MORAES, M. 2D Photography in facial asymmetry diagnosis. Int. J. Morphol., 33(4):1483-1486, 2015.

SUMMARY: The aim of this research was to find the relation between facial asymmetry and loss of continuity in median line of maxillary and mandibular teeth. A descriptive study was carried out in 18 subjects with facial asymmetry with surgical indication and observed at the Division of Oral and Maxillofacial Surgery of the Universidad de La Frontera. In all of them the grade of facial asymmetry and the relation in median line of the maxillary and mandibular teeth was evaluated using the $t$ student test considering $\mathrm{p}$ value $<0.05$; all the evaluations were executed using 2D photography of the face and tooth obtained with standardized conditions. In all the subjects we observed dental asymmetry with almost $7 \mathrm{~mm}$ from the median line; statistical relations between dental asymmetry and facial asymmetry. It is concluded that in subjects asking for dental asymmetry treatment a facial evaluation is necessary in view of the relation with skeletal facial deformity.

KEY WORDS: Photography; Facial asymmetry; Dental asymmetry. 


\section{REFERENCIAS BIBLIOGRÁFICAS}

Ettorre, G.; Weber, M.; Schaaf, H.; Lowry, J. C.; Mommaerts, M. Y. \& Howaldt, H. P. Standards for digital photography in craniomaxillo-facial surgery - Part I: Basic views and guidelines. $J$. Craniomaxillofac. Surg., 34(2):65-73, 2006.

Gateno, J.; Xia, J. J. \& Teichgraeber, J. F. Effect of facial asymmetry on 2-dimensional and 3-dimensional cephalometric measurements. J. Oral Maxillofac. Surg., 69(3):655-62, 2011.

Goulart, D. R.; Muñoz, P.; Olate, S.; de Moraes M. \& Fariña, R. No differences in morphological characteristics between hyperplastic condyle and class III condyle. Int. J. Oral Maxillofac. Surg., 44(10):1281-6, 2015.

Huentequeo-Molina, C.; Navarro, P.; Vásquez, B. \& Olate, S. Facial, Dental and Radiographic Analyses of Facial Normality. A Pilot Study in 29 Women. Int. J. Morphol., 31(1):150-5, 2013.

Kim, Y. H.; Sato, K.; Mitani, H.; Shimizu, Y. \& Kikuchi, M. Asymmetry of the sphenoid bone and its suitability as a reference for analyzing craniofacial asymmetry. Am. J. Orthod. Dentofacial Orthop., 124(6):656-62, 2003.

Maeda, M.; Katsumata, A.; Ariji, Y.; Muramatsu, A.; Yoshida, K.; Goto, S.; Kurita, K. \& Ariji, E. 3D-CT evaluation of facial asymmetry in patients with maxillofacial deformities. Oral Surg. Oral Med. Oral Pathol. Oral Radiol. Endod., 102(3):38290, 2006.

Muñoz, P.; Goulart, D. R.; Olate, S.; de Moraes, M.; Navarro, P. \& Fariña, R. Analysis of the Condyle, Articular Fossa and Mandibular Ramus in Subjects with Active Condylar Hyperplasia. Int. J. Morphol., 32(3):1064-8, 2014.

Nord, F.; Ferjencik, R.; Seifert, B.; Lanzer, M.; Gander, T.; Matthews, F.; Rücker, M. \& Lübbers, H. T. The 3dMD photogrammetric photo system in cranio-maxillofacial surgery: Validation of interexaminer variations and perceptions. $J$. Craniomaxillofac. Surg., 43(9):1798-803, 2015.

Nanda, V.; Gutman, B.; Bar, E.; Alghamdi, S.; Tetradis, S.; Lusis, A. J.; Eskin, E. \& Moon, W. Quantitative analysis of 3-dimensional facial soft tissue photographic images: technical methods and clinical application. Prog. Orthod., 16:21, 2015.

Olate, S.; Almeida, A.; Alister, J. P.; Navarro, P.; Netto, H. D. \& de Moraes, M. Facial asymmetry and condylar hyperplasia: considerations for diagnosis in 27 consecutives patients. Int. J. Clin. Exp. Med., 6(10):937-41, 2013 a.

Olate, S.; Cantín, M.; Alister, J. P.; Uribe, F.; Navarro, P.; Olate, G. $\&$ de Moraes, M. Relationship between condylar size and transverse facial asymmetry in subject with condylar hyperplasia. Int. J. Morphol., 31(3):937-41, 2013 b.
Olate, S.; Unibazo, A.; Almeida, A. \& de Moraes, M. Mandibular condylectomy revisited: technical notes concerning the use of an ultrasonic system. J. Oral Maxillofac. Surg., 72(3):481-4, 2014.

Proffit, W. \& Turvey, T. Assimetria dentofacial. En: Proffit, W.; White, R. \& Sarver, D. (Eds.). Tratamento Contemporáneo das Deformidades Dentofaciais. Porto Alegre, Artmed, 2005. pp.609-80.

Schaaf, H.; Streckbein, P.; Ettorre, G.; Lowry, J. C.; Mommaerts, M. Y. \& Howaldt, H. P. Standards for digital photography in cranio-maxillo-facial surgery--part II: additional picture sets and avoiding common mistakes. J. Craniomaxillofac. Surg., 34(6):366-77, 2006.

Song, W. C.; Koh, K. S.; Kim, S. H.; Hu, K. S.; Kim, H. J.; Park, J. C. \& Choi, B. Y. Horizontal angular asymmetry of the face in korean young adults with reference to the eye and mouth. $J$. Oral Maxillofac. Surg., 65(11):2164-8, 2007.

Supit, L. \& Prasetyono, T. A portable mirror stand for clinical facial photo documentation. Arch. Plast. Surg., 42(3):356-60, 2015 .

\author{
Dirección para Correspondencia: \\ Prof. Dr. Sergio Olate \\ Division of Oral and Maxillofacial Surgery \\ Universidad de La Frontera \\ Claro Solar 115, Oficina 404B \\ Temuco \\ CHILE
}

Email: sergio.olate@ufrontera.cl

Recibido : 12-06-2015

Aceptado: 25-08-2015 